cussed. Calculation of the neutrino flux at Earth resulting from individual active galactic nuclei is described along with the cosmological integration to find the contribution to the neutrino background. The prospects for observing both individual active galaxies and the diffuse background with the proposed neutrino telescopes are discussed. The results are compared to recent work by Stecker et al. (1992), Biermann (1992) and Sikora and Begelman (1992).

Chapter 7 summarises the work presented in this thesis and briefly discusses some of the other consequences of particle acceleration in active galaxies. The application of this work to other astrophysical objects is also briefly discussed.

\section{INVESTIGATIONS INTO RARE-EARTH SILICIDES AS INFRARED-SENSITIVE MATERIALS AND GENERALISED INFRARED ARRAY SYSTEMS}

\author{
Mark Helmut Unewisse \\ Physics Department, University of New South Wales, \\ Kensington NSW 2033 \\ April 1992
}

This thesis has investigated the physics of some of the rareearth silicide Schottky barrier contacts on silicon, in particular erbium silicide, and has attempted to determine whether they might be suitable for use as infrared detectors. To this end, yttrium silicide on $p$-type silicon and erbium silicide diodes on both $n$ and $p$-type silicon were produced. The rareearth silicides are of interest as they are the only known Schottky contacts that have a low barrier on n-type silicon and a high barrier on $p$-type silicon.

These diodes were then measured using current-voltage (I-V), current-voltage-temperature (I-V-T), and photoresponse techniques, for both forward and reverse bias. From these measurements the zero-bias and more fundamental flat-band barrier heights were determined for each diode produced. In the case of erbium silicide on $n$-type silicon, it was found that two types of diodes could be produced, either 'pitted' diodes with a barrier height of $\sim 0.35 \mathrm{eV}$ or 'smooth' diodes with a barrier height of $\sim 0.28 \mathrm{eV}$. The 'smooth' erbium silicide diodes were then extensively studied, as they held the greatest promise as infrared detectors.

Using photoresponse measurements, the quantum efficiency of the low barrier 'smooth' erbium silicide diodes was determined to be $\sim 0.52 \%$. Although small, this value is comparable to such established infrared detector materials as platinum silicide on $p$-type silicon, therefore showing erbium silicide to have great potential application as an infrared detector material.

The conduction mechanisms in erbium silicide Schottky diodes on $n$-type silicon have been studied over a temperature range of $25 \mathrm{~K}$ to $160 \mathrm{~K}$. Thermionic emission dominates carrier transport above $70 \mathrm{~K}$. Below this temperature, deviations are apparent in the zero-bias barrier height and ideality factor. However, the flat-band barrier height is shown to remain constant over the entire temperature range. The Fermi level is demonstrated to be pinned to the conduction band. A new quantity, the flat-band saturation current $\left(L^{f}\right)$ is defined and used in plots of $n * \ln \left(\mathrm{L}^{\mathrm{f}} \mathrm{T}^{2}\right)$ versus $1 / \mathrm{T}$, which give an excellent fit to the data over 28 orders of magnitude. From these plots the flat-band barrier height and the modified Richardson constant are obtained directly. This technique provides a completely self-consistent and more reliable way of obtaining these parameters than do previous methods. For low temperatures and low forward bias, recombination via tunnelling through surface states becomes the dominant conduction mechanism.

In addition to the work on the rare-earth silicides, a generalised imaging array control system has been developed in order to drive a wide range of infrared detector arrays. This system has been built for maximum flexibility, both in application and for future modification. The system is capable of driving almost any known imaging array.

\section{EVOLUTION FROM AGB STAR TO PLANETARY NEBULA}

Emanuel Vassiliadis

Mount Stromlo and Siding Spring Observatories, Australian

National University, Canberra ACT 2601

August 1992

This thesis presents a theoretical and observational study of stellar evolution for low to intermediate mass stars $(\sim 1-8$ $\mathrm{M}_{\odot}$ ), from the asymptotic giant branch (AGB) phase to the planetary nebula $(\mathrm{PN})$ phase.

In chapter 2 we have undertaken a series of theoretical calculations using the Mount Stromlo Stellar Structure Code to produce 22 stellar evolutionary sequences, complete from the main sequence phase through to the AGB phase. Models are calculated for initial masses $0.89,0.945,1.0,1.5,2.0$ $2.5,3.5$, and $5.0 \mathrm{M}_{\odot}$, and metallicities $0.016,0.008,0.004$ and $0.001 \mathrm{Z}_{\odot}$. These abundance and mass values were chosen to allow comparison with Galactic, and Magellanic Cloud stars. An important feature of these calculations is the inclusion of an empirical relationship between mass loss rate and pulsation period for AGB stars. The calculations lead naturally to a superwind phase, where the AGB is terminated by severe mass loss over the last 2-3 thermal pulse cycles. More precisely, the superwind phases occur towards the end of quiescent phases of helium shell flash cycles. The maximum AGB luminosities predicted from this work are in excellent agreement with those observed for Magellanic Cloud stars. The resulting initial-final mass relation predicts white dwarf masses $\sim 0.1 \mathrm{M}_{\odot}$ larger than the observational calibration of the relation.

In chapter 3 we continue the calculations presented in chapter 2 into the PN regime. Mass loss has also been included here, using an empirical formalism derived from observations of PN nuclei available in the literature. The evolutionary rates during the PN phase are examined, and are compared with those available in the literature. The calculations fall into two distinct groups; those where shell $\mathrm{He}$ burning is dominant, and those where shell $\mathrm{H}$ burning is dominant, when a star leaves the AGB phase. Low mass models are more likely to leave the AGB burning He, as the results in chapter 2 indicate the mass loss rate to be greatest immediately prior to a themal pulse.

In chapter 4 we examine the hydrodynamic behaviour of the winds produced during the AGB phase. A preliminary investigation was conducted over one complete shell flash cycle. At a thermal pulse event, an increase in wind speed drives a shell into the slower, pre-existing, quiescent wind. Following from the thermal pulse event, there is a significant luminosity decrease which allows matter to fall back towards the stellar surface, and which results in the formation of a hollow circumstellar shell. These characteristics may have already been noted in observations previously presented in the literature. 
In chapter 5 we present optical spectroscopy in the range $\lambda \lambda 3400-7900 \AA$ for a total of 52 planetary nebulae (PNe) in the Large Magellanic Cloud (LMC) and 18 in the Small Magellanic Cloud (SMC). This sample contains a large selection of objects of low luminosity, with measurements of line intensities down to $\sim 10 \%$ of $\mathrm{H} \beta$. Brighter objects have line intensities measured down to $\sim 2 \%$ of $\mathrm{H} \beta$. Reddening estimates from Balmer line ratios have been determined: unreddened and reddened line intensities are tabulated.

In chapter 6 we present echelle observations of 16 LMC PNe in the [O III] $\lambda 5007 \AA$ emission line, 10 of which are previously unobserved. An important property of these objects is that they lie far from the centre of the LMC and hence, their velocities provide strong constraints on the rotation of the LMC. These data have been used in conjunction with previously published data to redetermine the position angle of the kinematic line of nodes, $\Theta_{0}$, and the systemic Galactocentric velocity, $\mathrm{V}_{\mathrm{o}}$, of the LMC. After analysis of our data, combined with data available in the literature, we confirm the previous values derived from $\mathrm{PNe}$, where $\Theta_{0} \sim 170^{\circ}$ and $V_{0} \sim 40 \mathrm{kms}^{-1}$. The LMC is confirmed to consist of a single disk, and not a dual disk structure as originally proposed by Freeman, Illingworth and Oemler.

In chapter 7, we examine the effects of stellar effective temperature, and stellar and nebular metallicities, on the emission line fluxes used in the planetary nebular cosmic distance scale determinations. An absolute calibration of this distance scale is made by determining the metallicitycorrected, cut-off in the luminosity function of PNe. The distance modulus to the LMC is derived by fitting shell He burning PN nucleus evolutionary tracks (presented in chapter 3 ) to the observations. Distance modulii, relative to the LMC, are also derived for the SMC, Galactic Bulge, M31, and the Virgo cluster, after correcting the absolute PN nuclei luminosities for both stellar age and metallicity effects, and using the observed luminosity function cut-offs for these systems. In all the cases where Cepheid distances are known, a -0.14 mag zero-point offset is found with respect to the PN distance scale. It is concluded that the principle source of uncertainty in the PN distance scale is the intrinsic upper luminosity cut-off of the PN nuclei which, in turn, is critically dependent upon the stellar age (and therefore, mass) distribution, and the treatment of mass loss in the stellar evolutionary models.

\section{A SEARCH FOR HIGH-ENERGY GAMMA- RAYS FROM SUPERNOVA SN1987A}

\section{Liam Edwin Waldron \\ Department of Physics, University College, University of New \\ South Wales, Australian Defence Force Academy, \\ Canberra ACT 2600 \\ April 1992}

The Australian Defence Force Academy (ADFA) balloonborne gamma-ray astronomy telescope was flown successfully from Alice Springs Australia twice during 1987 and 1988 (flights 87-2-19 and 88-1-5) with the aim of measuring the gamma-ray flux, in the energy range 50 to $500 \mathrm{MeV}$, from Supernova SN1987A in the Large Magellanic Cloud. The two flights corresponded to day 55 and day 407 respectively of remnant evolution. The instrument was complemented by a hard X-ray proportional counter, designed and constructed by the Istituto di Astofisica Spaziale, CNR,
Frascati Italy, and sensitive to the 10 to $250 \mathrm{keV}$ energy range.

In this thesis, an account is given of the physical processes responsible for the production of gamma-rays in astrophysical environments and their relation to supernovae and cosmic-rays. A description is then given of the main features of the gamma-ray telescope and its principle of operation, the most important part of the telescope being a spark-chamber used to determine the direction of arrival of incident gamma-rays. Data obtained during each flight was recorded as spark-chamber tracks on photographic film. A detailed account of the methods of subsequent data reduction and analysis, as carried out by the author, are given. The principal results of this work were that 3- $\sigma$ upper limits to the gamma-ray flux from Supernova SN1987A of $2.2 \times 10^{-5}$ photons $\mathrm{cm}^{-2} \mathrm{~s}^{-1}$ and $3.4 \times 10^{-5}$ photons $\mathrm{cm}^{-2} \mathrm{~s}^{-1}$ were obtained for days 55 and 407 of remnant evolution respectively, these limits being somewhat lower than previously reported in the literature from a preliminary analysis of the data.

The above two upper limits are consistent with Supernova SN1987A being an atypical Type-II supernova. That is, the progenitor was a blue, rather than a red, supergiant. The limits are compared with theoretical predictions related to current models of gamma-ray emission from young Type-II supernovae.

\section{AN ULTRA-HIGH PRESSURE PROPORTIONAL COUNTER FOR HARD X-RAY ASTRONOMY}

\section{Zongnan $Y e$}

Department of Physics, University College, University of New

South Wales, Australian Defence Force Academy

Canberra ACT 2600

November 1992

This thesis describes the successful development of ultrahigh pressure proportional counters for balloon-borne hard $\mathrm{X}$-ray astronomy. The proportional counters were filled with argon/xenon at pressures up to $\sim 30 \mathrm{~atm}$. The properties of proportional counters filled at such pressures have been studied by the author in the laboratory. The spatial response of these counters to X-rays and charged particles, and the energy response to X-rays up to $1 \mathrm{MeV}$ have been analysed. Gas gain measurements using the charge collection technique and analysis of the subsequent data show that simple extrapolation from low pressures cannot explain the observed behaviour (e.g. the mobility of positive ions and quenching efficiency) of these counters at high pressures.

A hard X-ray telescope consisting of 32 such proportional counters filled at ultra-high pressures is being constructed, details of which are described. The sensitivity of this telescope for both continuum and narrow-line spectra is superb compared to contemporary balloon-and satellite-borne hard $\mathrm{X}$-ray detectors. Together with an imaging phoswich Anger camera, it is scheduled for launch from Alice Springs in November 1994.

An anticoincidence system for an X-ray detector, consisting of a combined passive and active shield, has been designed and constructed by the author, and has been flown on a balloon. The active shield, made of a plastic scintillator, has resulted in an additional reduction of $25 \%$ in the background registered at balloon altitudes. 